\title{
The Degree of Satisfaction of Medical Students Regarding Simulation Based Teaching Methods in Anesthesia and Intensive Care
}

\author{
Moldovan Cosmin ${ }^{1 *}$, Szederjesi Janos², Azamfirei Leonard ${ }^{3}$ \\ 1 Department of Histology, University of Medicine and Pharmacy of Tîrgu Mureș, Romania \\ 2 Department of Anesthesia and Intensive Care I, University of Medicine and Pharmacy of Tîrgu Mureș, Romania \\ 3 Department of Anesthesia and Intensive Care II and Emergency Medicine, University of Medicine and Pharmacy of Tîrgu Mureș, Romania
}

\begin{abstract}
Background: Anesthesia and Intensive Care is a teaching subject that arguably benefits the most from the use of simulation based methods in education. The availability of technically advanced complex simulators allows instructors to develop training scenarios that can be deeply integrated within the teaching curriculum. Aim: The present study aimed to assess whether the students undergoingAnesthesia and Intensive Caresimulation training are satisfied with the perceived educational outcome. Material and method: We carried out a retrospective transversal study in which we analyzed 256 feedback forms received from medical students between October 2014 and June 2015 . The forms contained 5 fixed questions that required rating a certain parameter with grades from 1 to 5 . Results: The simulation sessions used in Anesthesia and Intensive Care training were well perceived by students, over $90 \%$ of whom considered that these training session are useful from a professional and career development point of view. Conclusion: Based on the students' perception, simulation training sessions in Anesthesia and Intensive Care can be further developed and integrated with the clinical practical content of this subject.
\end{abstract}

Keywords: medical simulation, education, response

Received: 02 August 2015 / Accepted: 15 August 2015

\section{Introduction}

One of the most important moments in the history of medical simulation was the development of the first simulation mannequin, Resusci Annie, in 1960 [1]. This mannequin was used primarily in emergency medicine training, as it allowed for basic and medium-level resuscitation techniques to be performed. Full scale simulators have undergone a steady development in later years, and have evolved into very complex pieces of equipment that can be used to simulate challenging clinical scenarios.

The specifics of clinical training in Anesthesia and Intensive Care (AIC) are particular. Students are required not only to perform technical procedures, such as oro-tracheal intubation or ventilation, but also to display an integrative approach, using the knowledge they already have regarding various clinical conditions, and to be able to perform adequately in a team. The clinical setting needed for practicing and assessing these skills is not always readily available and, sometimes, can prove extremely challenging. Therefore, there is a risk of not conveying all the knowledge and skills to the students via practical training. On the other hand, the use of advanced simulators provides the opportunity to practice scenarios of a varying degree of difficulty in a riskfree environment [2]. The number and complexity of the scenarios that can be simulated is quite high, surpassing by far the possibilities of actual clinical teaching. That is not to say that actual clinical learning has become obsolete

* Correspondence to: Cosmin Moldovan

E-mail: cosmin.moldovan@outlook.com and should be replaced solely by simulation technologies. Rather, the use of simulation in AIC training should be regarded as a complementary tool in educating undergraduate students.

AIC simulations have been included in the curriculum of the University of Medicine and Pharmacy of Tîrgu Mureș since October 2013. The Center for Simulation and Practical Skills of the University offers students the opportunity to practice using one of the most advanced Human Patient Simulators available on the market. This simulation mannequin allows instructors to program scenarios ranging from simple to complex, in which automatic preset reactions of the simulated patient can be introduced, in order to improve the realistic feel of the simulations.

\section{Material and method}

We carried out a retrospective transversal study that consisted in analyzing the answers contained in 256 feedback forms distributed to and collected from $5^{\text {th }}$ year Medicine students undergoing simulation training between October 2014 and June 2015. The feedback forms were anonymous and were filled in by students without any external observation, so as not to influence the responses.

The questions contained in the feedback form were:

1. How interactive was the simulation you took part in?

2. Was the theme of the simulation easy to follow and understand?

3. Have you participated directly in the simulation?

4. Was the quality of the technical infrastructure satisfactory? 
5. From a professional standpoint, how useful do you think the simulation was?

The answers to the questions were presented as checkboxes with values ranging from 1 to 5 (1 meaning the lowest degree of satisfaction and 5 the highest one). All feedback forms contained identical questions and answer options for all subjects.

Statistical analysis was performed using Microsoft Excel and included only descriptive aspects.

\section{Results}

The answers to question 1 were as follows: $1-0.00 \%, 2$ $-0.78 \%, 3-2.34 \%, 4-15.23 \%$ and $5-81.64 \%$. In question 2 , the answers were 1 in $0.00 \%$ of the forms, 2 in $1.17 \%, 3$ in $7.81 \%, 4$ in $26.17 \%$ and 5 in $64.84 \%$, respectively. Question 3 had the following answer percentages, ranging from 1 to $5: 1.56 \%, 0.78 \%, 6.64 \%, 12.50 \%$ and $78.52 \%$, respectively. The answers to question 4 , arranged in the same order, were: $0.00 \%, 0.39 \%, 2.34 \%, 15.63 \%$ and $8.64 \%$. Finally, question 5 was answered with 1 in $0.00 \%$ of the forms, 2 in $0.39 \%, 3$ in $1.17 \%, 4$ in $4.69 \%$ and 5 in $93.75 \%$, respectively. An overview of the results is displayed in Figure 1.

\section{Discussion}

The responses given by the students in the feedback forms show a high degree of general satisfaction regarding the inclusion of simulation based scenarios in their training. The students who answered the questions in the feedback form had a varying degree of knowledge regarding Anesthesia and Intensive Care. Since the simulation scenarios take place during the entire academic year, students who undergo this training at the beginning of the first semester have a lower amount of information to rely on, whereas those who participate in the simulation later in the year possess more knowledge on this subject. Simulation sce- narios presented to the students included emergency cases related to Intensive Care.

The answers to the first question indicate that the degree of interactivity involved in the scenario the students had to undergo was perceived as high, and students experienced firsthand the results of their actions.

When analyzing the percentage of answers to the second question, a slight decrease in the overall proportion of the highest answer is apparent. This can be explained by the actual complexity of the situation the students had to handle during training. More specifically, the simulation protocol used in AIC training involves a fully developed clinical scenario. Within this scenario, the human patient simulator is programmed by the instructor to display signs and symptoms of a moderate-to-severe clinical emergency. Before entering the simulation room students are presented with basic data regarding the patient, and the instructors avoid conveying too much information that can divulge the diagnosis. Once they meet the simulated patient, students are required to undertake all necessary diagnostic steps for establishing the specific nature of the emergency in a real life timeframe. After a diagnosis is formulated, students need to assess and apply the correct therapeutic management in order to stabilize and treat the patient. For many students, this simulation is the first time they come in contact with a life-like emergency situation, and the stress induced by this could have contributed to the lower rate of the answers.

The answers to the third question are due to another particularity of the AIC simulation. Whereas during simulation based training sessions in other subjects all students can and are required to perform individually all the procedures, in AIC the participation is team based. In other words, in a specific group, each student is assigned a certain task to perform by the instructor (e.g. one student places the ECG leads, another one is responsible for administer-

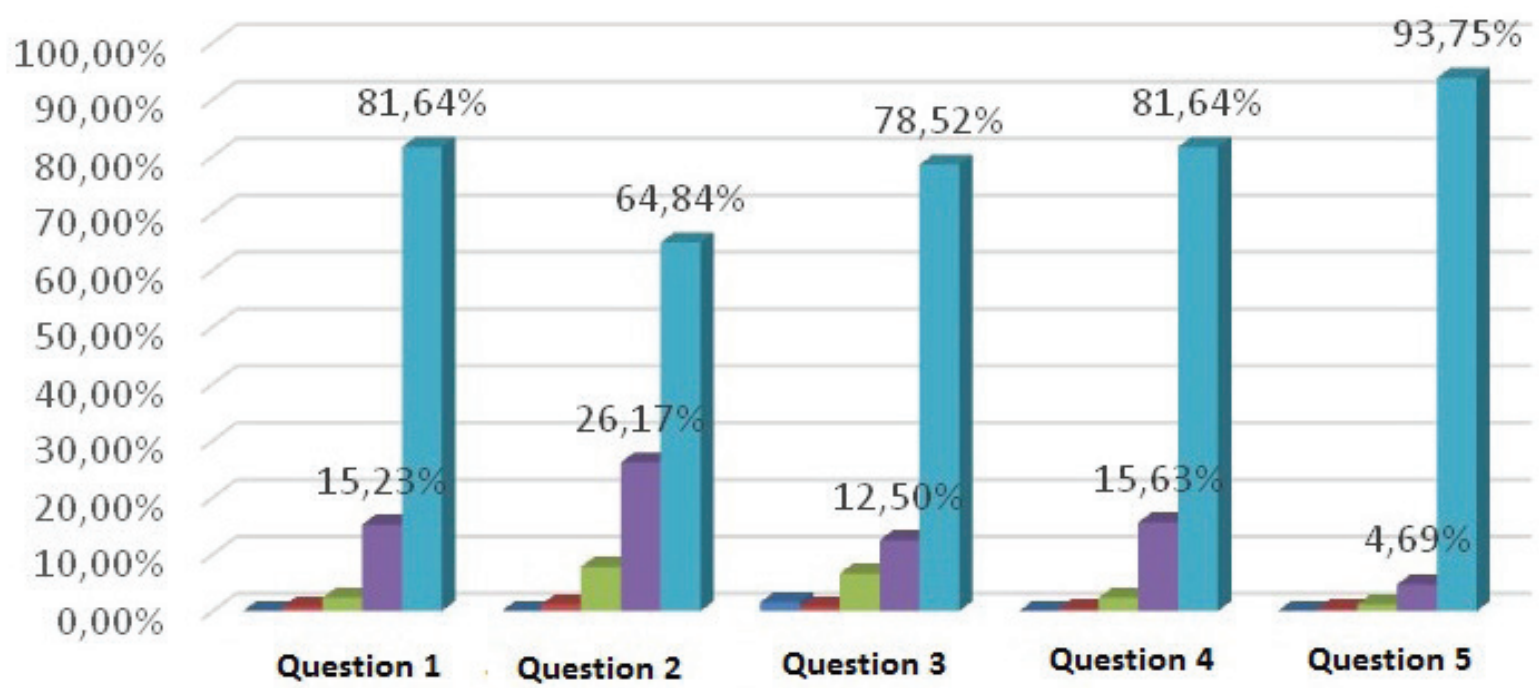

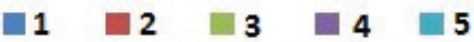

Fig. 1. Comparative evaluation of the answers to the questions in the feedback form. 
ing medication etc.) and therefore cannot carry out every maneuver required by the scenario. In addition, in each team there is a student whose task is exclusively to record all actions performed by the other students, for debriefing purposes, and therefore is prevented from actively taking part in the simulation.

The fourth question was answered positively by many students. One of the factors that prevented the percentage of maximum answers to be higher is precisely the one described before - one student is always taking notes, and the technical infrastructure is perceived as irrelevant with relation to the actual actions performed by this particular student.

Finally, from the answers to the fifth question it can be observed that almost all students consider simulation training to be an important or very important part of their future professional development.

Simulation based teaching is described in the literature as having a major positive impact on the outcome of medical education [3-5]. This outcome is generally assessed following the so-called Kirkpatrick's pyramid model [6]. Within this model, the first level is named "Reaction", and it involves evaluating how learners perceive the teaching methods applied in their training. This level was the rationale standing behind the present study. The next three levels ("Learning", "Behavior" and "Results") can be assessed over a longer period of time, in studies covering a larger student population and even medical professionals, and can provide an overview of the impact of simulation based medical training on professional performance.

\section{Conclusion}

Students perceive the introduction of simulation based training methods in the Anesthesia and Intensive Care curriculum as an overall positive learning experience. This positive reaction encourages us to further expand this particular area of medical education and to accelerate its curricular integration.

\section{Acknowledgment}

This paper was published under the frame of $\mathrm{Eu}$ ropean Social Fund, Human Resources Development Operational Programme 2007-2013, project no. POSDRU/159/1.5/S/136893.

\section{References}

1. Rosen KR. The history of medical simulation.Journal of Critical Care. 2008;23:157-166.

2. Morgan PJ, Cleave-Hogg D, Mcllroy J, Devitt JH. Simulation Technology. A Comparison of Experiential and Visual Learning for Undergraduate Medical Students.Anesthesiology. 2002;96:10-6.

3. O'Flynn S, Shorten G. Simulation in undergraduate medical education. European Journal of Anaesthesiology. 2009;26:93-95

4. Devitt JH, Kurrek MM, Cohen MM, Cleave-Hogg D. The Validity of Performance Assessments Using Simulation. Anesthesiology 2001;95:36-42.

5. Issenberg SB, McGaghie WC, Petrusa ER, Gordon DL, Scalese RJ. Features and uses of high-fidelity medical simulations that lead to effective learning: a BEME systematic review. Medical Teacher. 2005;27(1):10-28.

6. Sullivan GM. Deconstructing Quality in Education Research. J Grad Med Educ. 2011;3(2):121-124. 